\title{
Radar Observations of Venus in the Soviet Union in 1964
}

\author{
V. A. Kotelnikov \\ Institute for Radio Techniques and Electronics, Moscow, USSR
}

The radar observations of Venus were continued in the Soviet Union over the period of June 2-30, 1964. The measurements were carried out at a frequency of about $700 \mathrm{Mc} / \mathrm{s}$ using the same radar equipment that was used in 1961 and 1962 [Kotelnikov and Apraksin, 1962; Kotelnikov and Du Brovin, 1963]. A maser and parametric amplifier were used at the input of the receiver. The analysis of signals was performed from the magnetic tape by a 20-channel analyzer; the equivalent filter bandwidth of each channel was equal to $1.2 \mathrm{c} / \mathrm{s}$.

Two types of transmitter modulation were used, i.e., frequency-shift keying (FSK) and periodic linear frequency modulation similar to the method applied in 1962 [Kotelnikov and Du Brovin, 1963, 1964].

The radiated signal during frequency-shift keying had a form of alternating telegraphy pulses at two frequencies which differed either by $62.5 \mathrm{c} / \mathrm{s}$ or by $2000 \mathrm{c} / \mathrm{s}$. The duration of the pulses and intervals at each frequency were equal to 4.0 and $0.96 \mathrm{sec}$ respectively. This type of modulation was used for the purpose of investigating the spectrum of reflected signals and also for measurements of the radial velocity of Venus.

The linear frequency modulation was used for studying the angular-scattering law of radiowave reflection from the surface and also for measuring the distance to Venus. The frequency varied periodically according to a saw-tooth law [Kotelnikov and Du Brovin, 1964], and three operating modes were used: (1) deviation of $4 \mathrm{kc} / \mathrm{s}$ with a period of 1.024 sec; (2) deviation of $32 \mathrm{kc} / \mathrm{s}$ with a period of $8.192 \mathrm{sec}$; and (3) deviation of $32 \mathrm{kc} / \mathrm{s}$ with a period of $1.024 \mathrm{sec}$. The increase of deviation by a factor of eight gave a proportional improvement to the precision of distance measurement and range resolution for studying the angularscattering law from the surface.

The results of measurements of distance and radial velocity are given in figures $1 \mathrm{a}$ and $1 \mathrm{~b}$. Figure la shows the difference $\Delta r(\mathrm{~km})$ between the measured and calculated value of distance from the measuring station to the nearest point of the Venus surface. Figure lb shows the difference $\Delta V r(\mathrm{~cm} / \mathrm{sec})$ between the measured and calculated value of radial velocity of the Venus reflection center relative to the measuring station. The rms errors of measured values are shown by vertical lines.

The rms value of the equipment error during measurements of distance for one operating period which lasted for 5 min did not exceed $15 \mathrm{~km}$ until June 23 (with deviation of $4 \mathrm{kc} / \mathrm{s}$ ) and $2 \mathrm{~km}$ after June 23 (with deviation of $32 \mathrm{kc} / \mathrm{s}$ ). The error of the velocity measurement did not exceed $2.5 \mathrm{~cm} / \mathrm{sec}$.
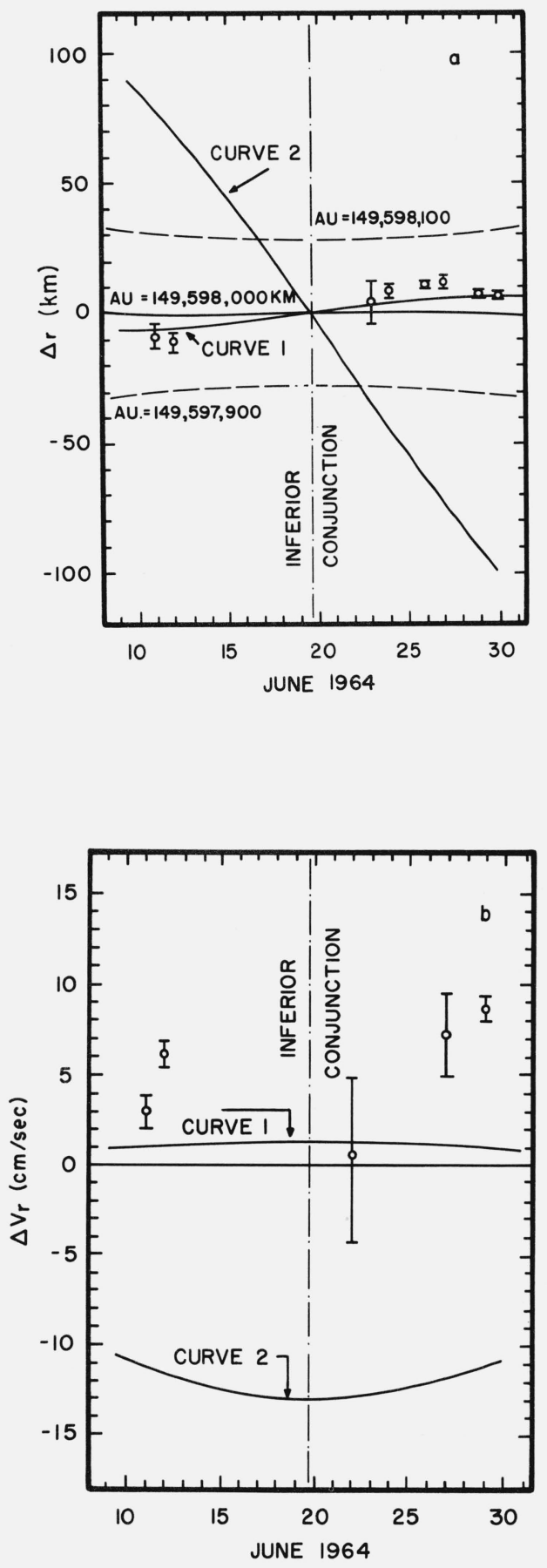

FIGURE 1. Variation of distance to Venus (a) and radial velocity of its motion (b) with reference to the calculated value. 
The calculations of the propagation time of signals and Doppler frequency shift assume the A.U. to be $149,598,000 \mathrm{~km}$, the velocity of light to be $299,792.5$ $\mathrm{km} / \mathrm{sec}$ and the radius of Venus to be $6100 \mathrm{~km}$. The propagation time of signals was calculated with an accuracy $\pm 5 \mu \mathrm{sec}$ and Doppler frequency $\pm 0.05 \mathrm{c} / \mathrm{s}$. The calculations of planet coordinates were carried out on the basis of the analytical theory of Newcomb with Duncombe corrections relative to the elements of Venus orbit, also taking into account the corrections made by Morgan relative to the elements of the EarthMoon system. In addition, account was taken of the small correction in orbital longitude apparently required by the 1961 and 1962 radar observations. The shift was determined [Kotelnikov and Du Brovin, 1963] and estimated to be $270 \mathrm{~km}$, which is equivalent to the increase of the heliocentric longitude of Venus by +0.52 sec. Smooth curve 1 corresponds to this shift given in figures $1 \mathrm{a}$ and $\mathrm{lb}$. In case an introduced correction to the shift was in fact absent, the experimental points should coincide with the smooth curve 2 . The dotted lines in figure la show how value $\Delta$ would be changed for values of the A.U. equal to $149,598,100$ and $149,597,900 \mathrm{~km}$.

The value of the A.U. as result of these measurements was equal to $149,598,000 \mathrm{~km}$, while a maximum error can be $\pm 400 \mathrm{~km}$ if we take into account possible systematic errors.

The rms values of systematic errors of the starting data recalculated to the A.U. are estimated by the following values: the velocity of light $-70 \mathrm{~km}$; the radius of Venus $-40 \mathrm{~km}$; the heliocentric coordinates of Venus- $100 \mathrm{~km}$; the influence of the medium in which a signal is propagated $-10 \mathrm{~km}$; other constants (the radius of the Earth, the relation of the mass of the Moon and the Earth and others) $-10 \mathrm{~km}$; the determination of the delay in the equipment $-5 \mathrm{~km}$. The total rms error equals to $130 \mathrm{~km}$.

The dependence of the energy distribution of Venusreflected signals on the distance $\Delta R$ relative to the nearest region of its surface to the Earth is given in figures $2 \mathrm{a}$ and $2 \mathrm{~b}$. The distribution shown in figure $2 \mathrm{a}$ is obtained during 27 periods of observation employing linear frequency modulation having a deviation of $4 \mathrm{kc} / \mathrm{s}$ and a period of $1.024 \mathrm{sec}$.

The first 11 columns represent an energy of signals reflected by the ring zones of the surface with the depth of $45 \mathrm{~km}$, the others with a depth of $150 \mathrm{~km}$. The distribution given in figure $2 \mathrm{~b}$ is obtained during 20 periods of observation using a frequency deviation of $32 \mathrm{kc} / \mathrm{s}$ and a period of $1.024 \mathrm{sec}$. The use of this deviation permitted a detailed investigation of the scattering law for the front region of the surface and allowed the energy from ring zones having a depth of $5.5 \mathrm{~km}$ to be measured. Using these data the energy dependence of the reflected signal power as a function of the angle of incidence shown in figure 3 by curve 1 was determined. A similar dependence obtained during the measurements made in 1962 is given for comparison in figure 3 by curve 2 .

A comparison of the results shows that a relative part of signal energy reflected from the nearest zone
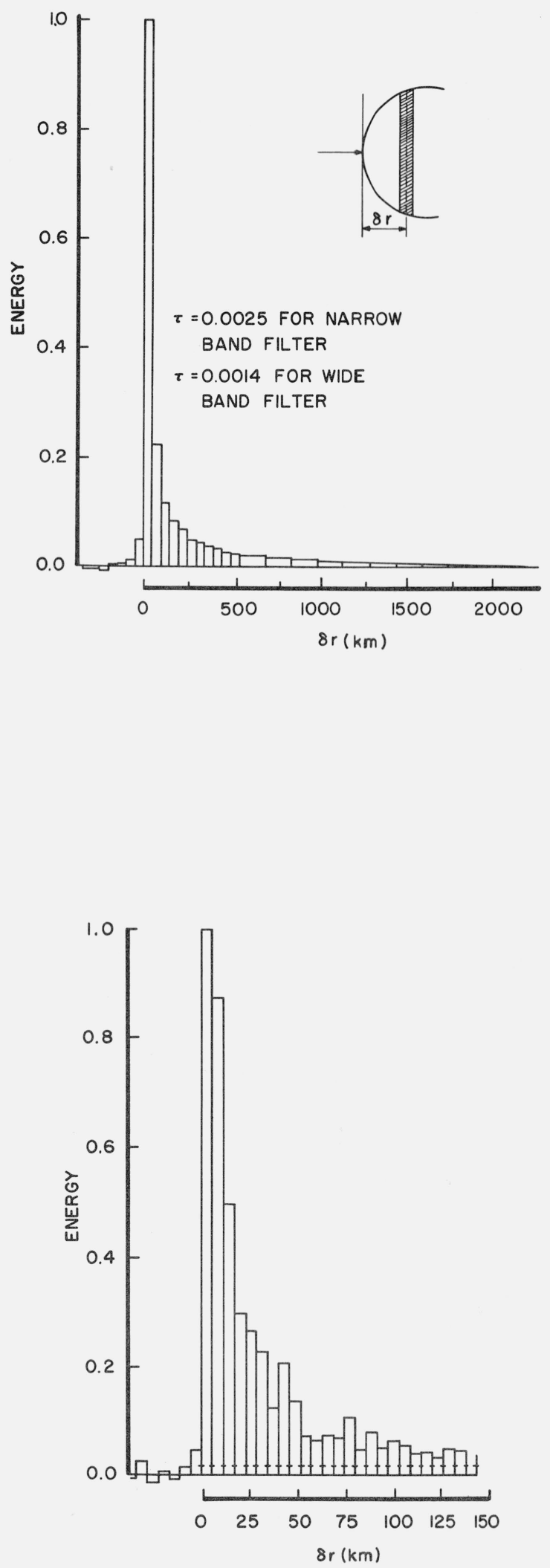

FIGURE 2. Distance distribution of energy of Venus reflected signals. Obtained during linear frequency modulation: (a) with deviation of $4 \mathrm{kc} / \mathrm{s}$, (b) with de. viation of $32 \mathrm{kc} / \mathrm{s}$. 


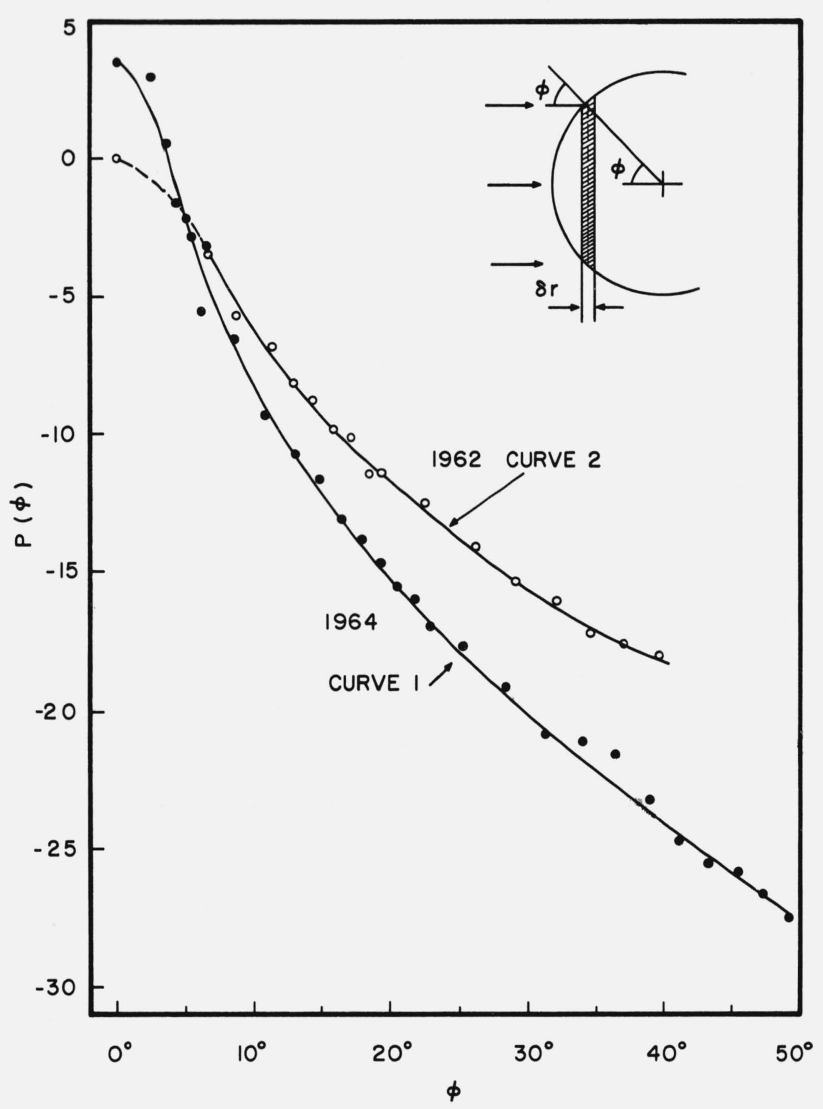

FigURE 3. Dependence of energy of reflected signals on the angle of incidence $\phi$; P-relation of energy density for angle to average density of the first zone with the depth of $45 \mathrm{~km}$.

of Venus surface in 1964 has greater value than in 1962. This can be explained by assuming that a smoother side of Venus was tuned toward the Earth during the 1964 radar observations.

The width of the Doppler spectrum of the reflected signal due to the rotation of Venus does not exceed $15 \mathrm{c} / \mathrm{s}$. The reflection coefficient [Kotelnikov and Du Brovin, 1964] of Venus measured according to the total energy of the received signal equals on the average 19 percent.

The energy of the central band of $1 \mathrm{c} / \mathrm{s}$ width is approximately two times less than the energy of the whole spectrum.

The spectra from separate observation days were used for the determination of the period of Venus rotation. For this purpose these spectra were compared with the spectra calculated for different periods of rotation on the basis of the energy distribution law given in figures $2 \mathrm{a}$ and $2 \mathrm{~b}$.

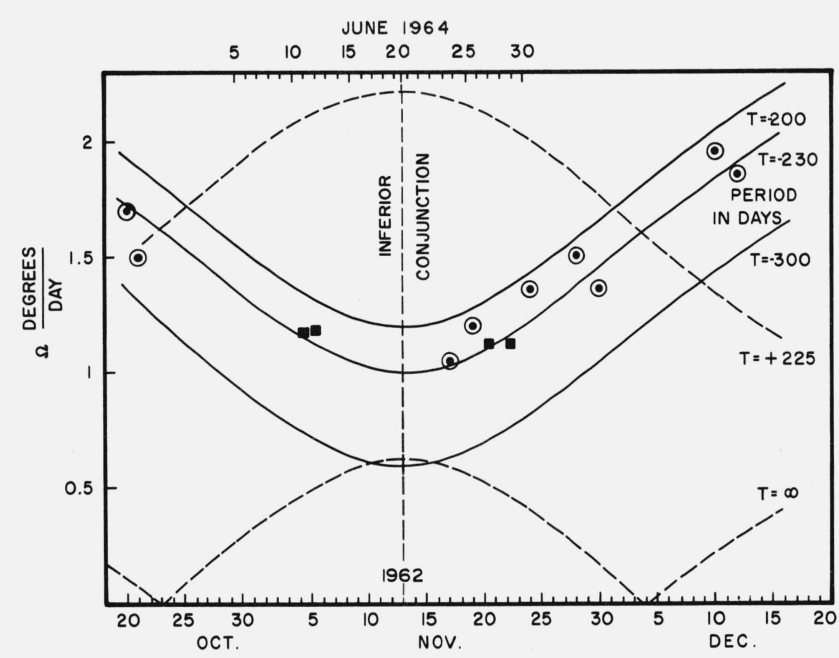

FIgURE 4. Determination of Venus rotation period according to the results of radar observations carried out in 1964 (squares) and in 1962 (circles); $\Omega$-angle velocity of Venus rotation with reference to the radar device.

The dotted lines show the calculated values $\Omega$ for direct rotation of Venus: the solid lines show the retrograde rotation.

The results obtained in 1964 do not contradict the conclusion concerning the retrograde rotation of Venus with a period of 200 to 300 days which were made during the radar observations of Venus in 1962 [Kotelnikov and Du Brovin, 1963]. The experimental results obtained in 1962 are shown in figure 4 by circles. The results obtained in 1964 are shown by squares. All these results obtained in 1964 are in a good agreement with each other and correspond to the retrograde rotation of Venus with a period of 230 days \pm 25 days.

The joint consideration of the results which determined the period of rotation in 1962 and 1964 shows that the orientation of Venus rotation axis is close to vertical with reference to the plane of the planet's orbit.

\section{References}

Kotelnikov, V. A., and L. V. Apraksin (1962), Radiolokatsionnaya Ustanovka, Ispolyovavshayaacya pru Radiolokatsii, Radiotekn. i Electron. 7, No. 11, 1851-1859.

Kotelnikov, V. A., and B. M. Du Brovin (1963), Radar observations of Venus in the Soviet Union in 1962, Dokl. Akad. Nauk 151, No. 3, 532-535.

Kotelnikov, V. A., and B. M. Du Brovin (1964), Supplement No. 9. 